\title{
FIGO Stage IIIA2 Ovarian Cancer
}

National Cancer Institute

\section{Source}

National Cancer Institute. FlGO Stage IIIA2 Ovarian Cancer. NCI Thesaurus. Code

C128091.

Ovarian cancer with microscopic, extrapelvic (above the brim) peritoneal involvement +/positive retroperitoneal lymph nodes. (FIGO, 2014) 